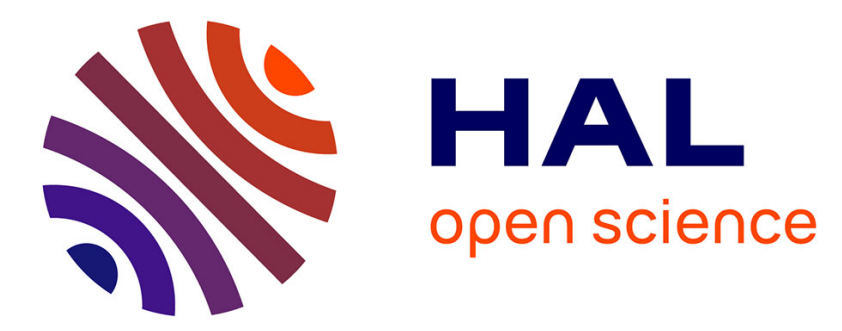

\title{
Abundance of rare and elusive species: Empirical investigation of closed versus spatially explicit capture-recapture models with lynx as a case study
}

Laetitia Blanc, Eric Marboutin, Sylvain Gatti, Olivier Gimenez

\section{- To cite this version:}

Laetitia Blanc, Eric Marboutin, Sylvain Gatti, Olivier Gimenez. Abundance of rare and elusive species: Empirical investigation of closed versus spatially explicit capture-recapture models with lynx as a case study. Journal of Wildlife Management, 2012, 77, pp.372 - 378. 10.1002/jwmg.453 . hal-03499359

\section{HAL Id: hal-03499359 \\ https://hal.science/hal-03499359}

Submitted on 25 Dec 2021

HAL is a multi-disciplinary open access archive for the deposit and dissemination of scientific research documents, whether they are published or not. The documents may come from teaching and research institutions in France or abroad, or from public or private research centers.
L'archive ouverte pluridisciplinaire HAL, est destinée au dépôt et à la diffusion de documents scientifiques de niveau recherche, publiés ou non, émanant des établissements d'enseignement et de recherche français ou étrangers, des laboratoires publics ou privés. 
4 Laetitia Blanc $^{1,2}$, Eric Marboutin ${ }^{2}$, Sylvain Gatti ${ }^{2}$, and Olivier Gimenez ${ }^{1}$

ABUNDANCE OF RARE AND ELUSIVE SPECIES: EMPIRICAL INVESTIGATION OF CLOSED VS.
SPATIALLY EXPLICIT CAPTURE-RECAPTURE MODELS WITH LYNX AS A CASE STUDY (1)

${ }^{1}$ Centre d'Ecologie Fonctionnelle et Evolutive, UMR 5175, campus CNRS, 1919 Route de Mende - F34293 Montpellier cedex 5

${ }^{2}$ Office National de la Chasse et de la Faune Sauvage - CNERA Prédateurs-Animaux déprédateurs, ZI Mayecin - F38610 Gières.

\section{Abstract}

Effective conservation and management require reliable monitoring methods and estimates of abundance to prioritize human and financial investments. Camera trapping is a non-invasive sampling method allowing the use of capture-recapture (CR) model to estimate abundance while accounting for the difficulty of detecting individuals in the wild. Here, we investigated the relative performance of classical closed CR models and spatially explicit CR models (SECR) that incorporate spatial information in the data. Using simulations, we considered four scenarios comparing low vs. high detection probability and small vs. large populations and confronted abundance estimates obtained from both approaches. Standard and SECR models both provided lowly biased abundance estimates but precision was improved when using SECR models. The associated confidence intervals also provided better coverage than their non-spatial counterpart. SECR models exhibit better statistical performance than standard closed CR models and allow producing sound management strategies through density maps of activity centers. To illustrate the comparison, we considered the Eurasian 
lynx (Lynx $\operatorname{lyn} x)$ as a case study and provided the first abundance estimates of a local population in France.

Keywords: abundance, relative bias, camera trapping, capture-recapture models, Lynx lynx, root mean square error, simulations, spatially explicit capture-recapture models.

\section{INTRODUCTION}

The presence of large carnivores - wolves, bears, lynxes, and wolverines - usually results in strong socio-cultural issues in all societies, Europe making no exception. These species share common features such as large territories and the need for a large mosaic of natural habitat and preys, potentially competing with human activities, e.g., hunting and livestock farming. Such conflicts, in combination with habitat loss, have led to local extinction of large carnivores in many areas. While almost extinct at the beginning of the 20th century in many European countries, large carnivores have slowly recovered via reintroduction or natural recolonization through dispersal.

In this context, the Bern convention (1979), the European Habitats Directive (1992) as well as the International Union for Conservation of Nature (IUCN) Red list provided specific indexes and rules to assess the conservation status of species and to help checking how management decisions could meet the conservation requirements. Abundance was defined as one of the key estimates needed in assessing species' status and is the state variable of interest in most ecological research and monitoring programs involving management and conservation of animal populations (Nichols and MacKenzie 2004). Indeed, reliable estimates of population size are essential to evaluate conservation and wildlife management programs such as reintroduction programs. However, large carnivores are difficult to monitor since they are 
elusive, living at low densities over wide areas and usually solitary and mostly nocturnal.

Exhaustive counts are therefore often expensive, time consuming and sometimes impractical.

In order to assess population trends in elusive and wide-ranging population, non-invasive survey methods have been increasingly used over the last decade. In particular, cameratrapping methods combined with capture-recapture (CR) modeling have become a standard tool to estimate carnivores' abundance while accounting for detectability less than 1 (e.g., tigers Panthera tigris: Karanth et al. 2006, Karanth and Nichols 1998; ocelots Felis pardalis: Trolle and Kéry 2003; snow leopards Uncia uncia: Jackson et al. 2006; jaguars Panthera onca: Silver et al. 2004). Standard CR models usually assume geographical closure (no movement in or off the sampling grid). However, this assumption is often violated, especially for mammals with large home range. Another major assumption of these models is that no individual within the sampled area has a zero probability of being captured. To deal with these issues, an alternative approach known as spatially-explicit CR modeling (SECR) was recently developed (Royle and Young 2008, Borchers and Efford 2008). This method has been applied to a large number of taxa (e.g., birds: Efford 2004, Borchers and Efford 2008, Efford et al. 2009a; cetaceans: Marques et al. 2010; stoats: Efford et al. 2009b; bears: Obbard et al. 2010 and lizards: Royle and Young 2008). Here, the probability of detection for each trap is modeled as a function of distance between a latent variable, the individual activity center (equivalent to the home range center), from which animals move randomly, and the camera trap where they have been captured. This model does not rely on the assumption of geographic closure by accounting for the fact that animals move and that detection probability depends on their center of activity (Gardner et al. 2009).

In this paper, our objectives were twofold. First, we aimed at evaluating the relative performance of SECR methods vs. conventional non-spatial CR models in estimating 
abundance in the context of carnivore conservation. Most of the studies assessing bias in spatial models compared abundance estimates using real datasets rather than simulated data, hence the impossibility to infer bias and precision (e.g., Gardner et al. 2009). Recent papers dealing with SECR models and simulations (Efford 2004, Royle and Young 2008, Borchers and Efford 2008, Efford 2011) focused on the performance of different methods to estimate density (e.g., nested subgrid vs. inverse prediction, likelihood-based vs. Bayesian methods) but did not formally compare SECR and non-spatial models. Therefore, we carried out a simulation study with several scenarios comparing low vs. high detection probability and small vs. large populations to quantify the performances of parameter estimates using both SECR and non-spatial models. We also suggested how the simulations results could be used to improve the trapping design when necessary. Second, we used the two methods to analyze a real dataset from a camera-trapping experiment with the Eurasian lynx (Lynx lynx) in the French Jura Mountains. This population originates from reintroductions in Switzerland in the 70's. Although listed as a species of Least Concern given its wide range (IUCN, 2001), habitat loss, prey depletion, and poaching are still regarded as potential threats. Up to now, the main monitoring program for lynx in France was based on indirect signs (i.e., tracks, scat, hair, and other signs) collected by a network of volunteers (state employees, hunters, naturalists, farmers, and mountain guides). While the use of indirect signs is often the most effective and least expensive method for estimating the distribution of carnivores, the resulting estimates of population parameters such as abundance are often approximate. Camera-trapping monitoring has recently been initiated in France in order to monitor lynx population and evaluate the conservation status of a population where problematic interactions between hunters and lynx exist. We provided the first estimate of Lynx abundance for this French population. Finally, recommendations are provided for the conservation of elusive species, with an emphasis on large carnivores and their monitoring. 
MATERIAL AND METHODS

\section{$97 \quad$ Simulation study design}

We considered that our population was demographically and geographically closed (i.e. no birth, death, immigration or emigration during the sampling period) to apply CR models to estimate abundance. Lynx are long-lived animals (Sunquist and Sunquist 2002) and the camera-trap sampling period was made short enough so that no deaths or births were assumed to occur during this period. In addition, the trapping session was timed outside the dispersal period for subadults.

In order to compare the performance of the standard vs. the SECR methods in estimating abundance, we simulated 100 datasets with a particular spatial organization. We considered four scenarios comparing low vs. high detection probability and small vs. large populations. These scenarios were used to evaluate relative bias in parameter estimates, the precision and the coverage of $95 \%$ confidence and credible intervals (CI hereafter for Bayesian credible intervals or Frequentist confidence intervals indistinctively). Each dataset was created using the traps configuration from the monitoring of the lynx in the study area (see case study below) but we did not use any constraints to mimic lynx behavior simulating the datasets. The number of capture occasions was set to $\mathrm{k}=15$ and the actual population size to $\mathrm{N}=10$ or $\mathrm{N}=$ 50 depending on the scenario. The simulations were based on the SECR model formulation. We simulated $N$ individual activity centers using their coordinates. Then, we evaluated whether we could a posteriori reliably estimate from the model the actual number and location of activity centers we had simulated. We proceeded to the simulation in two steps, first a point process component that describes the spatial distribution of the centers of activity, second an observation process component that makes the connection between the detection of an individual and its center of activity given the spatial distribution of traps. 
121 We assumed a fixed and known number of activity centers $\mathrm{s}_{\mathrm{i}}$ (similar to home range centers)

122 with geographic coordinates $\mathrm{s}_{\mathrm{i}}=\left(\mathrm{s}_{\mathrm{xi}}, \mathrm{s}_{\mathrm{yi}}\right)$ for each individual $i(i=1, \ldots, \mathrm{N})$ of the population.

123 We assumed that these centers of activity were uniformly distributed over a region S, an

124 arbitrary polygon containing the trapping array.

125

$\mathrm{s}_{\mathrm{i}} \sim \operatorname{Uniform}(\mathrm{S}) \quad$ (Eq. 1)

In order to simulate capture histories we assumed that the probability of each individual to be captured was a function of the distance between its activity center and the trap.

The detection probability of an individual at a given trap was a decreasing function of the distance from the activity center to that trap: the further the activity center was from the trap, the less likely the animal was exposed to capture. Thus, we first defined a distance matrix $D_{i, j}$ as the Euclidean distance between every activity centers $i$ and trap $j$ :

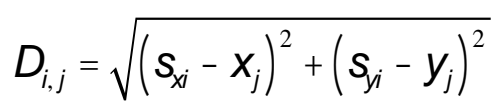

134 Second, we modeled the exposure of each individual as a function of distance and two other 135 parameters:

$$
E 0_{i, j}={ }_{0} \exp \left(D_{i, j}^{2} /\right)
$$

137 where $\lambda_{0}$ is the baseline encounter rate, i.e. the expected number of captures of individual $i$ at $138 \operatorname{trap} j$ during a sampling occasion when an individual's activity center $\mathrm{s}_{\mathrm{i}}$ is located precisely at trap $j$, and parameter $\sigma$ (in $\mathrm{km}$ ) controls the shape of the distance function, reflecting how fast 
140 the exposure decreases with distance. The greater $\sigma$ is, the faster the exposure decreases with 141 distance.

143 If an individual $i$ is exposed to trap $j$, we assumed a capture probability $\mathrm{p}_{\mathrm{i}, \mathrm{j}}$. The distance

144 function allows the development of the capture process model. The increase of the exposure

145 to traps translates into an increase of the capture probability and was modeled with an 146 exponential function:

147

$$
p_{i, j}=1 \exp \left(E 0_{i, j}\right) \quad \text { (Eq. 4) }
$$

148 We assigned two different values for $\lambda_{0}(0.03$ and 2$)$ and one value to $\sigma(1.5)$ depending on the 149 scenario. All combinations of all levels of $\mathrm{N}, \lambda_{0}$ and $\sigma$ were tested resulting in 4 scenarios. For 150 each scenario and each simulated dataset, we constructed the distance matrix $D_{i, j}$ between the 151 simulated activity centers and the traps location. The distance matrix was used to estimate for 152 each individual a per trap capture probability $\mathrm{p}_{\mathrm{i}, \mathrm{j}}$. Then, we performed a binomial trial with 153 parameters $\mathrm{N}$ and $\mathrm{p}_{\mathrm{i}, \mathrm{j}}$ to determine whether the individual was captured or not. Since detection 154 is not perfect, only $n$ out of the $\mathrm{N}$ total individuals from the population were detected. We compiled for each of the $\mathrm{J}$ traps the number of occasions $\mathrm{K}$ an individual $i$ was detected. Thus,

156 for each trap and each individual, a code number ranging from 0 to $\mathrm{K}$ indicated how many

157 occasions each individual was captured. These count histories were used to fit SECR models.

158 Finally, we analyzed the capture histories of the $n$ individuals under the standard and SECR 159 models.

\section{Model formulation}


162

163

164

165

We first calculated abundance estimates by accounting for detection probabilities using standard CR models. We considered different sources of variation in capture probabilities. In addition to a model with no variation in the detection probability (model $\mathrm{M}_{0}$ ), we considered behavioral responses to trapping (model $\mathrm{M}_{\mathrm{b}}$ ), differences in capture probabilities over time (model $\mathrm{M}_{\mathrm{t}}$ ), while the most complex models included among-individual heterogeneity in capture probabilities (model $\mathrm{M}_{\mathrm{h}}$ ) (Otis et al. 1978, Williams et al. 2002). In addition, we considered four models that were combinations of these sources of variation (Models $\mathrm{M}_{\mathrm{bh}}$, $\mathrm{M}_{\mathrm{th}}, \mathrm{M}_{\mathrm{tb}}$, and $\mathrm{M}_{\mathrm{tbh}}$ ). For each simulated dataset, the Akaike's Information Criterion (AIC) was used to select the model that best described our dataset (Burnham and Anderson 2002). These analyses were achieved via maximum likelihood with the R package Rcapture (Baillargeon and Rivest 2007).

\section{b) SECR model implementation using a Bayesian approach}

Each camera trap reflected the location of capture, which, in turn, provided insight into the activity centers coordinates of each lynx. The SECR model has the advantage to incorporate spatial heterogeneity while estimating abundance (Royle et al. 2009a, Royle et al. 2011, Royle et al. 2009b). More specifically, the SECR model makes explicit the distinction between a) a latent component for the spatial point process of the (unknown) location of the activity centers (Eq. 1) and b) an observation component that describes how the observed data arise from the point process (Eq. 4).

We adopted a Bayesian approach (McCarthy 2007) to fit the SECR model. It made the analysis convenient as the activity centers are treated as random effects that are relatively easy to deal with in the Bayesian framework (King et al. 2009). The Bayesian approach combines the likelihood with prior probability distributions of the parameters to obtain the posterior distribution of the parameters of interest based on Bayes' theorem. We used Markov Chain 
186

187

188

189

190

191

192

193

194

195

196

197

198

199

200

201

202

203

204

205

206

207

Monte Carlo (MCMC) methods to simulate observations from the posterior distributions.

Regarding priors for parameters, we considered that we did not have any information about the spatial distribution of the activity centers of the simulated individuals thus we assumed they were uniformly distributed over S. We chose a Uniform $(0,15)$ distribution for $\sigma$ and we assigned a $\operatorname{Gamma}(0.1,0.1)$ distribution to $\lambda_{0}$.

To obtain an estimate of abundance, we used a data augmentation approach (Royle and Young 2008). We augmented the data set with 100 individuals and we associated to every individual a latent indicator $\mathrm{z}_{\mathrm{i}}$. The encounter histories of the 100 individuals initially contained only zeroes. Some of these individuals were not captured during the intensive camera trapping but belonged to the population. The $\mathrm{z}_{\mathrm{i}}$ indicator reflects the probability $\psi$ of an individual to be a member of the population. We assumed a Uniform $(0,1)$ prior for $\psi$ that we added as an additional layer to our model. We defined $z_{i}$ as a binary variable equals to 0 when the individual $i$ is not a member of the population and 1 otherwise.

The abundance $\mathrm{N}$ was obtained as a derived parameter by adding all the presence indicators $\mathrm{Z}_{\mathrm{i}}$. These analyses were implemented in WinBUGS (Spieghalter et al. 2003) called from $\mathrm{R}$ using package R2WinBUGS (Sturtz et al. 2005).

c) Evaluating the performance of the two methods

We evaluated the performance of the standard CR models and the SECR models by comparing the abundance estimates of the two methods used on each 100 datasets simulated to the true value of abundance. As a result, we were able to quantify the potential bias in parameter estimates obtained for both models. We looked at the relative bias in $\hat{N}$, the estimator of $\mathrm{N}$, calculated as $(\mathrm{E}[\hat{N}]-\mathrm{N}) / \mathrm{N}$ which can be approximated as the average over the 100 iterations of the difference between the estimated abundance under the model 
210 calculated the Root Mean Square Error $(\mathrm{RMSE})$ as $\sqrt{E\left(([\hat{N}]-N)^{2}\right)} \quad \sqrt{{ }_{i=1}^{100}\left(\hat{N}_{i} \quad N\right)^{2} / 100}$.

211 A low RMSE is characteristic of a good trade-off between low bias and high variance. Finally,

212 we looked at the $95 \%$ confidence interval coverage by determining and averaging over all

213 simulations whether the interval contained the true value.

\section{Eurasian lynx in French Jura Mountains}

215 Lynx is a solitary nocturnal species, living in forested areas. It can be individually identified 216 based on the photographs of their unique pelage patterns (e.g., Zimmermann and

217 Breitenmoser 2007). Our study area was located in the French department of Jura. A $480 \mathrm{~km}^{2}$ 218 zone was considered in the southern center of the Jura department between the Vouglans lake 219 and the southern border of Doubs department. This study area was delimited using knowledge on lynx habitat and forest continuity. In order to maximize detectability, several steps were followed: 1) Camera traps were set at optimal locations (on game path, hiking trail, forest road) based on previous signs of lynx presence and on local knowledge; 2) In theory, all

223 individuals should have a non-null detection probability to use standard capture-recapture models (Karanth and Nichols 1998). It is not necessary for SECR models (Royle et al. 2009a). Thus, the study area was divided into a grid of $2.7 \mathrm{~km} \times 2.7 \mathrm{~km}$ cells (Zimmermann et al. 2007) where one of two cells was sampled, leading to 33 cells sampled from February to April 2011. This grid size and sampling design ensure that at least one camera trap site is set

228 in each potential lynx home range; 3) At each trapping site, two camera traps with infrared trigger mechanism were set in order to photograph both flanks of the animal allowing a high level of confidence in individual identification. Date, time and location of each photographic capture of a lynx were recorded; 4) Camera traps were checked weekly to change memory 
232

233

234

235

236

237

238

239

240

241

242

243

244

245

246

247

248

249

250

251

252

253

254

255

cards and batteries. The sampling period was divided into 15 occasions, one occasion being defined as 4 successive trap nights. The results of the SECR model were used to build a density map of the lynx activity centers. For each of the MCMC iterations, we plotted the centers of activity of the individuals belonging to the population $\left(\mathrm{z}_{\mathrm{i}}=1\right)$ on successive layers. For every layer, we divided the region $\mathrm{S}$ into squares of $500 \times 500 \mathrm{~m}$ then we calculated the mean number of activity centers falling into each square. $\mathrm{R}$ and WinBUGS codes are available on request from the first author.

\section{RESULTS}

\section{Simulation study to compare spatial vs. non-spatial models}

For each scenario and each simulated dataset, we reported the abundance posterior median estimate and its $95 \%$ credible interval for the SECR model and the abundance point estimate with its $95 \%$ confidence interval from the non-spatial model (Fig. 1). Scenario A represented a small population with a low detection probability mimicking the Eurasian lynx dataset. Both models similarly slightly overestimated abundance: the non-spatial model displayed a relative bias of 0.096 and the SECR model relative bias was 0.121. Scenario B represented a large population with a low detection probability. The non-spatial model clearly underestimated the population size with a relative bias of -0.08 while the SECR model slightly underestimated it with a -0.016 relative bias. Scenario $\mathrm{C}$ corresponded to a small population with a high detection probability. For most datasets, the non-spatial model provided estimates close to the actual abundance (relative bias around 0.007) but with large confidence intervals and the SECR model also provided unbiased estimates (relative bias around -0.02) and small credible intervals. Finally, scenario D represented an ideal situation with a large population and a high detection probability. The non-spatial model slightly overestimated abundance (relative bias = 0.026) while the SECR model provided values close to the actual abundance (relative bias = 
0.0002). RMSE clearly revealed that the SECR model provided a better balance between bias and variance for all scenarios than the non-spatial model. With regard to CI coverage, the confidence interval of the non-spatial model included the true abundance value in only 73 to 78 out of the 100 simulated datasets depending on the scenario. The credible interval of the SECR model included the true value in 92 to 99 datasets (Table 1). Credible intervals of the SECR model provided better coverage than confidence intervals as provided by standard closed CR models.

\section{Lynx case study}

Data were collected between February and April 2011 from 33 trap sites resulting in 1980 trap nights. One site was found effective during less than $50 \%$ of the trapping nights. It was removed from the analysis reducing the theoretical effort to 1816 effective trapping nights. The study provided an encounter history for 9 individuals that were photographed on 14 of the 32 trap sites. Individuals were captured on up to six different sites and the maximum distance moved by one individual between captures was $27.6 \mathrm{~km}$. Model selection ranked $\mathrm{M}_{\mathrm{h}}$ incorporating individual heterogeneity in capture probability as the best model followed by $\mathrm{M}_{0}$ assuming constant capture probability. Average estimated detection (over individuals) was 0.14 and the estimated abundance using $\mathrm{M}_{\mathrm{h}}$ was 12 individuals (95\% CI 7.14-20.27). For the SECR model, the baseline encounter rate at a given camera $\left(\lambda_{0}\right)$ was 0.05 photographs occasion $^{-1}(95 \%$ CI $0.03-0.15)$ while the movement parameter $\sigma$ was estimated to $1.45(95 \%$ CI 0.16-0.58). The abundance was estimated to 12.04 individuals (95\% CI 9.0-18.0).

Posterior estimates of activity centers' locations of the 9 individuals photographed are shown in Fig. 2. There was a lot of spatial variation in the location of estimated activity centers, most of them being concentrated in the center and in both south-eastern and western corners of the trap array. 


\section{DISCUSSION}

281

Information on wildlife population responses and dynamics are essential complements to the human dimensions, habitat, and ecosystem functioning that go into conservation planning and monitoring (Mills 2007). Using the Eurasian lynx as a case study, we demonstrated how cutting-edge analytical methods could be used to estimate and infer abundance of a rare and elusive species using sound monitoring protocols. This is a crucial step when implementing any conservation strategy so as to be able e.g. to characterize the population status before any action is undertaken, and re-evaluate it once management has been engaged.

\section{Comparison of abundance estimates from spatial vs. non-spatial models}

Albeit the difference in the relative bias between the non-spatial and the SECR model is trivial, the RMSE and the CI coverage both support the conclusion that the SECR model provides better estimates of abundance. Indeed, our simulations have highlighted that for scenario A, mimicking the lynx dataset, and scenario C, abundance estimates should be used with caution since the spatial model tended to overestimate the actual abundance while the non-spatial model appeared to be closer to the real abundance value. The positive relative bias may be caused by the proportion of individuals that move out or partially out of the trapping array creating an inflated estimate of abundance. Nevertheless, confidence and credible interval coverage and RMSE revealed that the SECR model performed best whatever the scenario we considered. For the other scenarios, B and D - representing a large population with respectively a low and high detection probability - abundance estimates were closer to the actual value when using the spatial model. The three deviation indices (relative bias, RMSE and interval coverage) supported this conclusion. 
302

303

304

305

306

307

308

309

310

311

312

313

314

315

316

317

318

Spatially-explicit CR modeling is an emerging analytical tool that has mainly been used to estimate densities because it does not rely on the assumption of geographic closure (Efford 2004). Obbard et al. (2010) and Gray and Prum (2012) evaluated the performance of the SECR models while estimating density by comparing density estimates using SECR with those obtained from conventional approach in which the effective survey area is estimated using a boundary strip width. SECR models were recommended in both studies but they could not infer bias since the actual density was unknown. Efford (2004) and Borchers and Efford (2008) assessed the performance of SECR by simulating data from a regular grid of trap.

They used alternative methods for fitting the spatial detection model, that is inverse prediction and maximum likelihood while the current study used data augmentation and MCMC (Royle and Young 2008; Royle et al. 2009a, b). Regardless of the method, the importance of spatial nature of the sampling process in capture probability modeling is clearly supported by our findings. Modeling the capture probability also avoids substantial bias in estimating abundance. By making capture probability a function of both the location of the activity centers and their distance from the camera traps, SECR models allow efficient use of spatial information contained in CR data.

We acknowledge that we could not cover all possible scenarios in our simulations. In particular, our results were obtained for scenarios that did not account for specificities of the species biology, such as sex-related differences in home range size for instance (Sollmann et al. 2011). Furthermore, we did not take into account the importance of traps configuration that can have large effects on the number of individuals detected. In our study, the traps were placed mainly on trails because lynx use the easiest way to go from one location to another.

Further work is needed to determine the optimal number and location of traps in order to minimize the human and financial costs of fieldwork while maximizing the precision of 
abundance estimates. Simulation studies like the one we have conducted here may help in that purpose. SECR models allow the incorporation of variables affecting detection probability hence providing managers the opportunity to modify cameras distribution to improve capture success (Royle et al. 2011).

Non-invasive sampling methods such as camera trapping or molecular tracking are commonly used to monitor elusive and wide-ranging populations of large carnivores, as neither of them requires physical captures. These methods can provide estimates of population parameters, like population size, dispersal distance, population growth rate (Marescot et al. 2011), survival (Cubaynes et al. 2010), recruitment and immigration rate (Karanth et al. 2006). They seemed particularly relevant for the Eurasian lynx whose individual coat patterns allow the identification on photographs that could be used with capture-recapture models to estimate abundance and density. Furthermore, the advantage of camera-trapping for estimating abundance is that it requires only a single sampling session, in other words repeated sampling is not required (Efford et al. 2009). However, this technique requires reliable photographs from which individuals can be univocally identified, otherwise risking bias in population size estimates (overestimation if two photographs belonging to the same individual are considered as two different individuals, underestimation if two photographs of different individuals are wrongly considered as a single individual). The issue of misidentification error has recently received interest (Yoshizaki et al. 2009; Morrison et al. 2011).

\section{Management implications}

With rare and elusive species, we recommend caution when using standard or even spatiallyexplicit capture-recapture models since commonly few data are available. Even though previous studies have demonstrated the utility of non-invasive sampling methods (e.g., Petit and Valière 2006) and the analysis of data collected through CR techniques (e.g., Rees et al. 
350

351

352

353

354

355

356

357

358

359

360

361

362

363

364

365

366

367 Baillargeon, S., and L. P. Rivest. 2007. Rcapture: Loglinear models for capture-recapture in R.

368 Journal of Statistical Software.

369 Borchers, D. L. and M. G. Efford. 2008. Spatially explicit maximum likelihood methods for

2011) when few data are available, the confidence and credible intervals still remain large. A preliminary simulation study is useful to determine which factors affect abundance estimates (number of camera traps and their location in particular). To help in this objective, we provide $\mathrm{R}$ code to reproduce our simulation exercise and adapt it for one's own purpose (See

Supplementary Information). Pending these precautions, spatially explicit CR models provide useful information that can be used to produce sound management strategies for carnivores. In particular, the density map of the posterior locations of activity centers could be confronted to livestock attacks distribution maps to determine whether correlations exist between hotspots of attacks on livestock and pools of lynx centers of activity. This might help to predict potential conflicts between human activities and predators.

\section{ACKNOWLEDGMENTS}

L. Blanc was supported by Labex Centre Méditerranéen de l'Environnement et de la Biodiversité (CeMEB) and University of Montpellier 2. We thank the staff from the French National Game and Wildlife Agency, the Forest National Agency and the "federation départementale des chasseurs" and the volunteers who collected the photographs during the camera-trapping session.

\section{LITERATURE CITED} capture-recapture studies. Biometrics 64:377-385. 
371 Burnham, K. P., and D. R. Anderson. 2002. Model selection and inference: a practical 372 information theoretic approach. Second edition. Springer-Verlag, New York, New York, 373 USA.

374 Cubaynes, S., R. Pradel, R. Choquet, C. Duchamp, J. M. Gaillard, J. D. Lebreton, E. Marboutin, 375 C. Miquel, A. M. Reboulet, C. Poillot, P. Taberlet, and O.Gimenez. 2010. Importance of 376 accounting for detection heterogeneity when estimating abundance: the case of French 377 wolves. Conservation Biology 24:621-626.

378 Efford, M. G. 2004. Density estimation in live-trapping studies. Oikos 106:598-610.

379 Efford, M. G. K., D. Dawson, and D. L. Borchers. 2009a. Population density estimated from 380 locations of individuals on a passive detector array. Ecology 90:2676-2682.

381 Efford, M. G., D. L. Borchers, and A. E. Byrom. 2009b. Density estimation by spatially 382 explicit capture-recapture: likelihood-based methods. Pages 255-269 in D. L.

383 Efford, M. G. 2011. Estimation of population density by spatially explicit capture-recapture analysis 384 of data from area searches. Ecology 92:2202-2207.

385 Thompson, E. G. Cooch, M. J. Conroy, editors. Modeling demographic processes in marked 386 populations. Springer, New York.

387 Gardner, B., J. A. Royle, and M. T. Wegan. 2009. Hierarchical models for estimating density 388 from DNA mark-recapture studies. Ecology 90:1106-1115.

389 Gray, T. N. E. and S. Prum. 2012. Leopard density in post-conflict landscape, Cambodia: 390 Evidence from spatially explicit capture-recapture. Journal of Wildlife Management $391 \quad 76: 163-169$.

392 Jackson, R. M., J. D. Roe, R. Wangchuk, and D. O. Hunter. 2006. Estimating snow leopard 393 population abundance using photography and capture-recapture techniques. Wildlife Society 394 Bulletin 34:772-781. 
395 Karanth, K. U., J. D. Nichols, N. S. Kumar, and J. E. Hines. 2006. Assessing tiger population 396 dynamics using photographic capture-recapture sampling. Ecology 87:2925-2937.

397 Karanth, K. U., and J. D. Nichols. 1998. Estimation of tiger densities in India using 398 photographic captures and recaptures. Ecology 79:2852-2862.

399 King, R., B. J. T. Morgan, O. Gimenez, and S. P. Brooks. 2009. Bayesian analysis of population 400 ecology. CRC Press.

401 Knapp, S. M., B. Craig, and L. P. Waits. 2009. Incorporating genotyping error into non402 invasive DNA-based mark-recapture population estimates. Journal of Wildlife Management $403 \quad 73: 598-604$.

404 Marescot, L., R. Pradel, C. Duchamp, S. Cubaynes, E. Marboutin, R. Choquet, C. Miquel, and 405 O. Gimenez. 2011. Capture-Recapture population growth rate as a robust tool against 406 detection heterogeneity for population management. Ecological Applications 21:2898-2907. 407 Marques, T. A., L. thomas, S. W. Martin, D. K. Mellinger, S. Jarvis, R. P. Morrissey, C. 408 Ciminello, and N. DiMarzio. 2010. Spatially explicit capture recapture methods to estimate 409 minke whale abundance from data collected at bottom mounted hydrophones. Journal of 410 Ornithology in press.

411 McCarthy, M. A. 2007. Bayesian methods for ecology. Cambridge University Press, 412 Cambridge, UK.

413 Mills, L. S. 2007. Conservation of Wildlife Populations: Demography, Genetics, and 414 Management. Blackwell Publishing, Malden, Massachusetts, USA.

415 Morrison, T. A., J. Yoshizaki, J. D. Nichols, and D. T. Bolger. 2011. Estimating survival in 416 photographic capture-recapture studies: overcoming misidentification error. Methods in 417 Ecology and Evolution 2:454-463.

418 Nichols, J. D. and J. D. MacKenzie. 2004. Abundance and conservation biology. Animal 419 biodiversity and conservation 27.1:437-439. 
420 421

422

423

424 Petit, E., and N. Valiere. 2006. Contributed Papers: Estimating population size with

425

426

427

428

429

430

431

432

433

434 Royle, J. A., J. D. Nichols, K. U. Karanth, and A. M. Gopalaswamy. 2009. A hierarchical

Obbard, M. E., E. J. Howe, and C. J. Kyle. 2010. Empirical comparison of density estimators for large carnivores. Journal of Applied Ecology 47:76-84.

Otis, D. L., K. P. Burnham, G. C. White, and D. R. Anderson. 1978. Statistical inference from capture data on closed animal populations. Wildlife Monograph 62:1-135. noninvasive Capture-Mark-Recapture data. Conservation Biology 20:1062-1073.

Rees, S. G., A. E. Goodenough, A. G. Hart, and R. Stafford. 2011. Testing the effectiveness of capture mark recapture population estimation techniques using a computer simulation with known population size. Ecological Modelling 222:3291-3294.

Royle, J. A. and K. V. Young 2008. A hierarchical model for spatial capture recapture data. Ecology 89:2281-2289.

Royle, J.A., K. U. Karanth, A.M. Gopalaswamy, and N.S. Kumar. 2009. Bayesian inference in camera trapping studies for a class of spatial capture-recapture models. Ecology 90:32333244.

435 model for estimating density in camera-trap studies. Journal of Applied Ecology 46:118-127.

436 Royle, J. A., A. J. Magoun, B. Gardner, P. Valkenburg, and R. E. Lowell. 2011. Density 437 estimation in a wolverine population using spatial capture-recapture models. The Journal of 438 Wildlife Management 75:604-611.

439 Silver, S. C., L. E. T. Ostro, L. K. Marsh, L. Maffei, A. J. Noss, M. J. Kelly, R. B. Wallace, H. 440 Gómez, and G. Ayala. 2004. The use of camera traps for estimating jaguar Panthera onca 441 abundance and density using capture/recapture analysis. Oryx 38:148-154.

442 Sollmann, R., M. M. Furtado, B. Gardner, H. Hofer, A. T.A. Jácomo, N. M. Tôrres, and L. 443 Silveira. 2011. Improving density estimates for elusive carnivores: Accounting for sex- 
444 specific detection and movements using spatial capture-recapture models for jaguars in 445 central Brazil. Biological Conservation 144:1017-1024.

446 Spieghalter, D. J., A. Thomas, and N. Best. 2003. WinBUGS, Version 1.4, User Manual. 447 Sturtz, S., U. Ligges, and A. Gelman. 2005. R2WinBUGS: A Package for Running WinBUGS 448 from R. Journal of Statistical Software 12:1-16.

449 Sunquist, M., and F. Sunquist. 2002. Wild Cats of the World. University of Chicago Press, 450 Chicago, USA.

451 Trolle, M., and M. Kéry. 2003. Estimation of ocelot density in the Pantanal using Capture452 Recapture analysis of camera-trapping data. Journal of Mammalogy 84:607-614.

453 Williams, B. K., J. D. Nichols, and M. J. Conroy. 2002. Analysis and management of animal 454 populations : modeling, estimation, and decision making. Academic Press, San Diego, 455 California, USA.

456 Yoshizaki, J., K. H. Pollock, C. Brownie, and R. A. Webster. 2009. Modeling misidentification 457 errors in capture-recapture studies using photographic identification of evolving marks. 458 Ecology 1:3-9.

459 Zimmermann, F., and U. Breitenmoser. 2007. Potential distribution and population size of the 460 Eurasian lynx Lynx lynx in the jura Mountains and possible corridors to adjacent ranges. 461 Wildlife Biology 13:406-416.

\section{CAPTIONS}

Table 1. Summary of the statistical performance of the non-spatial and SECR models. The

466 RMSE is the Root Mean Square Error and CI is either the 95\% confidence (non-spatial 467 model) or the $95 \%$ credible (SECR model) interval. 


\begin{tabular}{ccccc}
\hline & Scenario & Relative bias & RMSE & Cl coverage \\
\hline \multirow{5}{*}{ Non-spatial model } & A & 0,10 & 4,00 & $75 \%$ \\
& B & $-0,08$ & 9,38 & $76 \%$ \\
& C & 0,01 & 1,03 & $78 \%$ \\
& D & 0,03 & 5,08 & $73 \%$ \\
\hline \multirow{3}{*}{ SECR model } & A & 0,12 & 2,39 & $97 \%$ \\
& B & $-0,02$ & 5,49 & $92 \%$ \\
& C & $-0,02$ & 0,47 & $99 \%$ \\
& D & 0,00 & 0,89 & $96 \%$ \\
\hline
\end{tabular}

469

470 Fig. 1. Comparison between abundance estimates obtained from non-spatial models vs. SECR

471 models according to four scenarios (low detection probability (A,B) vs. high detection

472 probability $(\mathrm{C}, \mathrm{D})$ and small population size (A,C) vs. large population size (B, D)). With grey

473 dots and lines we displayed respectively estimates and confidence intervals for the non-spatial

474 model. With black asterisk and black lines we displayed posterior means and 95\% credible

475 intervals obtained with the SECR model. The vertical dashed line indicates the actual value of 476 abundance.

477 Fig. 2. Map of posterior density of lynx activity centers in French Jura department.

478 Specifically, the map shows E[N(i) | data], where N(i) is the number of activity centers

479 located in pixel i. Colors code for the estimated number of activity centers in each $500 \times$

$480500 \mathrm{~m}$ pixel; triangles indicate mean activity center location for identified individuals; dots

481 indicate camera trap locations; black ones indicate locations where lynx were photographed

482 and grey ones where no lynx was captured. 
484

Fig. 1

485

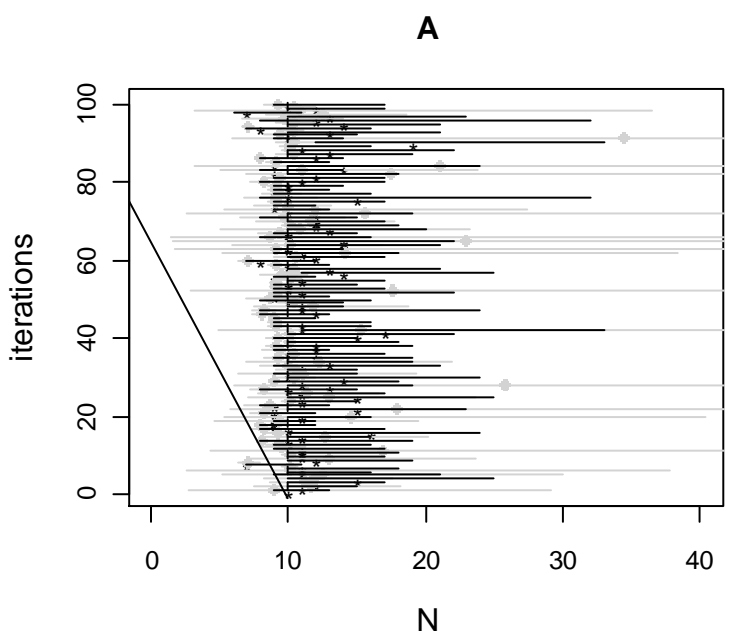

D

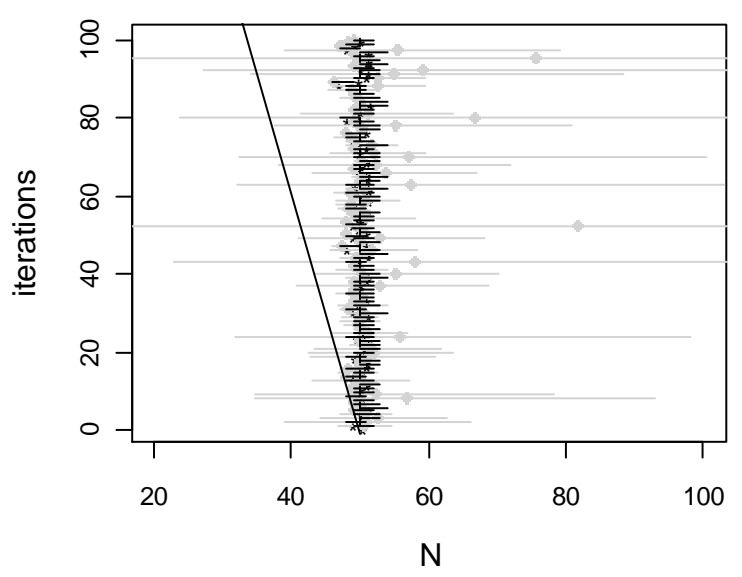

B

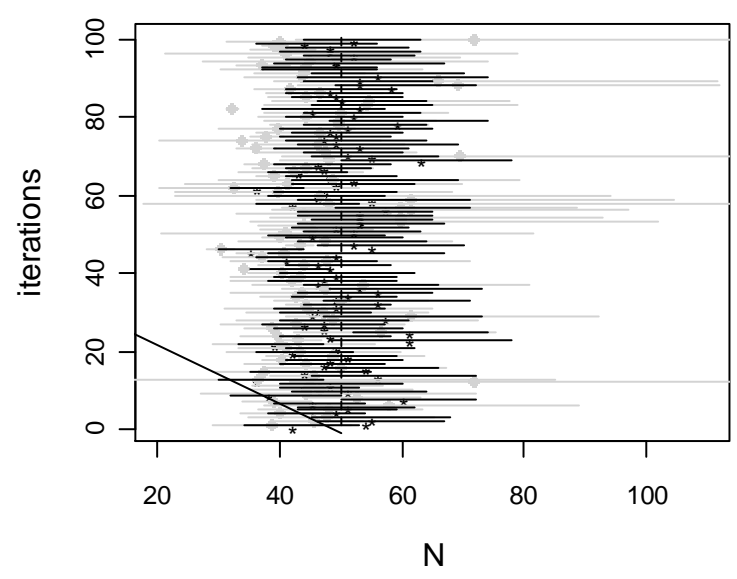

C

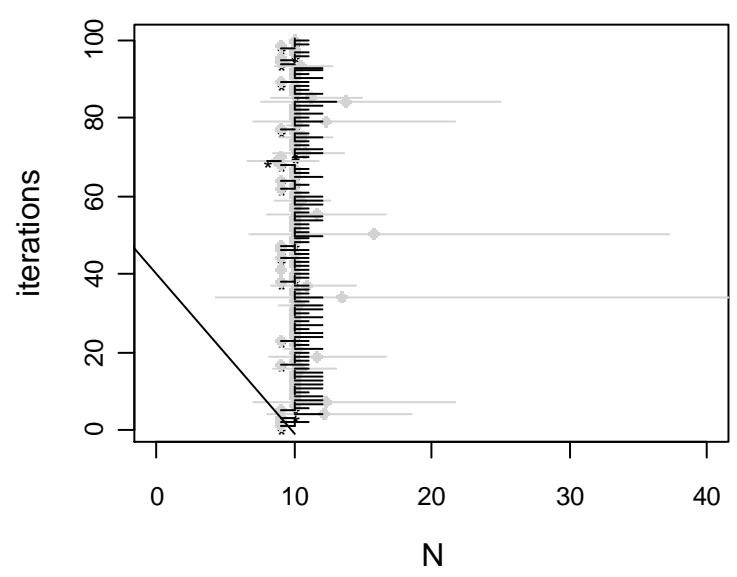

486

487 
Fig. 2

489

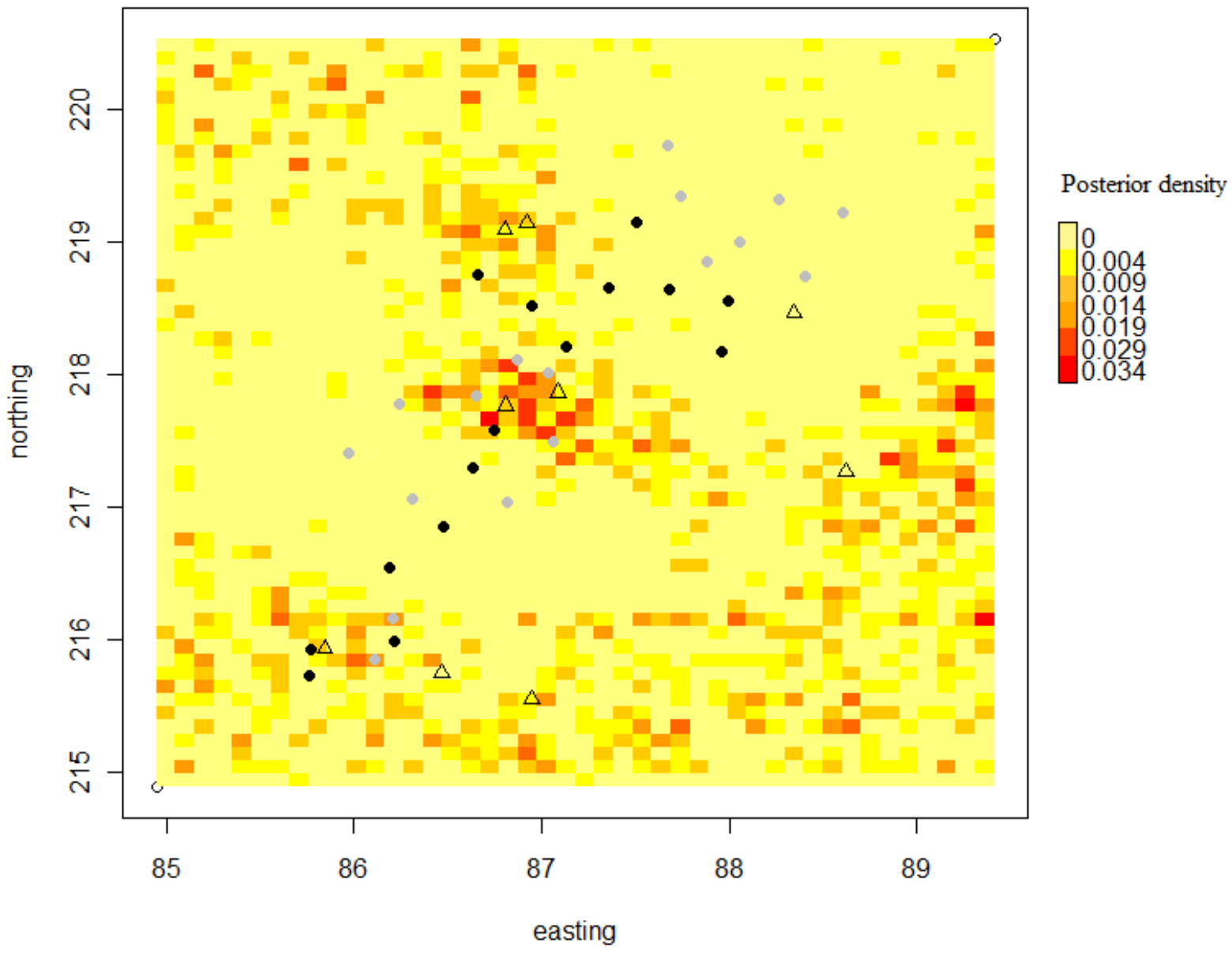

